\title{
Flujo venoso pulmonar con ecocardiografía transesofágica intraoperatoria durante cirugía cardíaca
}

\author{
Intraoperative pulmonary vein flow with transesophageal echocardiography \\ during cardiac surgery
}

\author{
Silvina Longo 1,3,*, Pablo Merlo², Cecilia Acosta³, Walter González4, Juan Macedo Romoacca4, María Carolina Cabrera Schulmeyer ${ }^{5}$ \\ Anestesióloga Hospital Privado Universitario de Córdoba. Argentina. \\ Docente Universidad de Buenos Aires. Asociación Argentina de Ultrasonografía Crítica (ASARUC. Argentina. \\ Anestesióloga Hospital Privado de la Comunidad de Mar Del Plata. Argentina. \\ Residentes de Anestesiología Hospital Provincial Castro Rendón de Neuquén. Argentina. \\ Anestesióloga Universidad de Valparaíso. Valparaíso, Chile.
}

Fecha de recepción: 12 de agosto de 2021 / Fecha de aprobación: 18 de octubre de 2021

\begin{abstract}
Doppler flow analysis of the pulmonary veins with transesophageal echocardiography is very useful during cardiac surgery. It can be performed in most patients due to the proximity of the probe to the left atrium. It is data that allows us to evaluate the filling of the left heart and evaluate the impact of mitral disease. In this review we analyze the formation of Doppler waves and highlight the importance of their analysis during cardiac surgery.
\end{abstract}

Key words: Transesophageal echocardiography, pulmonary veins Doppler, diastolic function.

\section{RESUMEN}

El análisis del flujo Doppler de las venas pulmonares con ecocardiografía transesofágica es de gran utilidad durante cirugía cardíaca. Se puede realizar en la mayoría de los pacientes debido a la proximidad de la sonda a la aurícula izquierda. Es un dato que nos permite evaluar el llenado del corazón izquierdo y evaluar la repercusión de la enfermedad mitral. En esta revisión analizamos la formación de las ondas con Doppler y destacamos la importancia de su análisis durante cirugía cardíaca.

Palabras clave: Ecocardiografía transesofágica, doppler de venas pulmonares, función diastólica.

\section{Introducción}

I flujo de la vena pulmonar (VP) mediante doppler de onda pulsado es útil para comprender las consecuencias hemodinámicas de los trastornos que afectan la función cardíaca. Se conocen claramente cómo los trastornos valvulares, miocárdicos, pericárdicos, congénitos, del ritmo y la conducción alteran el perfil doppler de la VP[1]. El registro óptimo de la señal de flujo de PV y el análisis adecuado de la dirección, velocidad y duración de las formas de onda doppler distinguen los patrones de flujo normal de los anormales y relacionan los patrones de flujo anormales con trastornos específicos que afectan particularmente al corazón izquierdo.

Durante el intraoperatorio debemos responder preguntas acerca del estado del corazón izquierdo en el menor lapso de tiempo posible.

El objetivo de esta revisión es proporcionar un análisis integral del papel del doppler de la VP en la práctica quirúrgica con ecocardiografía transesofágica (ETE) y destacar su utilidad en diversos trastornos cardiovasculares en el perfil de flujo de VP. 
Conocer el perfil del doppler VP mejora nuestra capacidad de diagnóstico y promueve nuestra comprensión de la fisiopatología de varios trastornos cardíacos durante la cirugía.

\section{Anatomía venosa pulmonar}

Por lo general, las VP son cuatro, superior e inferior derecha e izquierda que drenan hacia la aurícula izquierda con orificios separados. Sin embargo, esta anatomía está presente en solo el $70 \%$ de los individuos[2]. Las venas superior e inferior derecha se extienden por detrás de la vena cava superior y la aurícula derecha y se conectan medialmente a las caras superior y posterior de la aurícula izquierda, respectivamente, cerca del tabique interauricular. Las venas superior e inferior izquierda corren por delante de la aorta descendente y se conectan a la pared lateral de la aurícula izquierda (Figura 1). En el 30\% restante de los individuos, se observan variantes anatómicas de las VP, incluidas las venas conjuntas que se unen proximales a la aurícula izquierda, dando como resultado un ostium único[2].

\section{Ecocardiografía transesofágica}

Debido a la proximidad del transductor, la ETE proporciona una óptima visualización de las VP, permitiendo tomar muestras del flujo de diferentes venas[3].

Las VP se obtienen de la ventana medio esofágica (ME), y se facilita por el uso de doppler color. Tanto las venas superiores como la VP inferior izquierda (VPII) corren oblicuas o casi verticales al plano de imagen de ETE, mientras que la VP inferior derecha (VPID) discurre más horizontalmente y se localiza más posteriormente. Las venas superiores son más fáciles de visualizar y, con la VPII, son las más adecuadas para interrogar utilizando doppler de onda pulsada debido a una mejor alineación con el haz de ultrasonido. Los orificios de las venas superior e

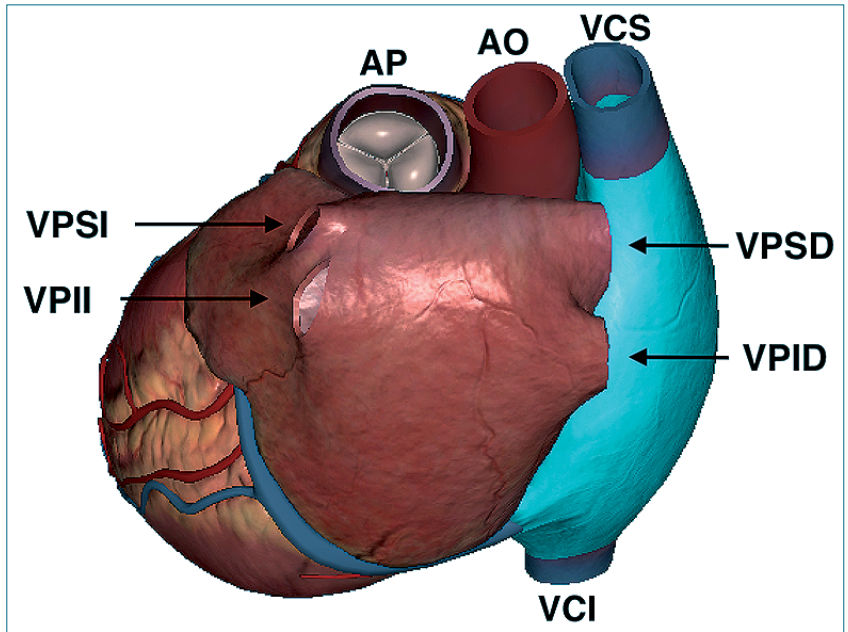

Figura 1. Relación de las venas pulmonares superiores e inferiores derecha e izquierda, drenando en aurícula izquierda, con otras estructuras cardíacas. (venas cavas superior e inferior: VCS, VCl; aorta: $\mathrm{AO}$; pulmonar: $\mathrm{AP})$.

inferior están muy próximos entre sí. Cuando se identifica un PV superior, un ligero movimiento hacia adelante de la sonda y/o un cambio mínimo en el ángulo del transductor generalmente permite la visualización de la vena inferior correspondiente[10]. Por tanto, es posible visualizar las VP superior e inferior, derecha o izquierda, en el mismo plano de imagen. El volumen de la muestra debe colocarse aproximadamente a $1 \mathrm{~cm}$ dentro de la vena desde el ostium para el interrogatorio Doppler de onda pulsada.

La VPSI a menudo se visualiza bien adyacente al apéndice de la aurícula izquierda (AI) con cierta angulación del transductor $30^{\circ}$ a $60^{\circ}$ (Figura 2a)[8]. Más angulación oblicua (90-120)

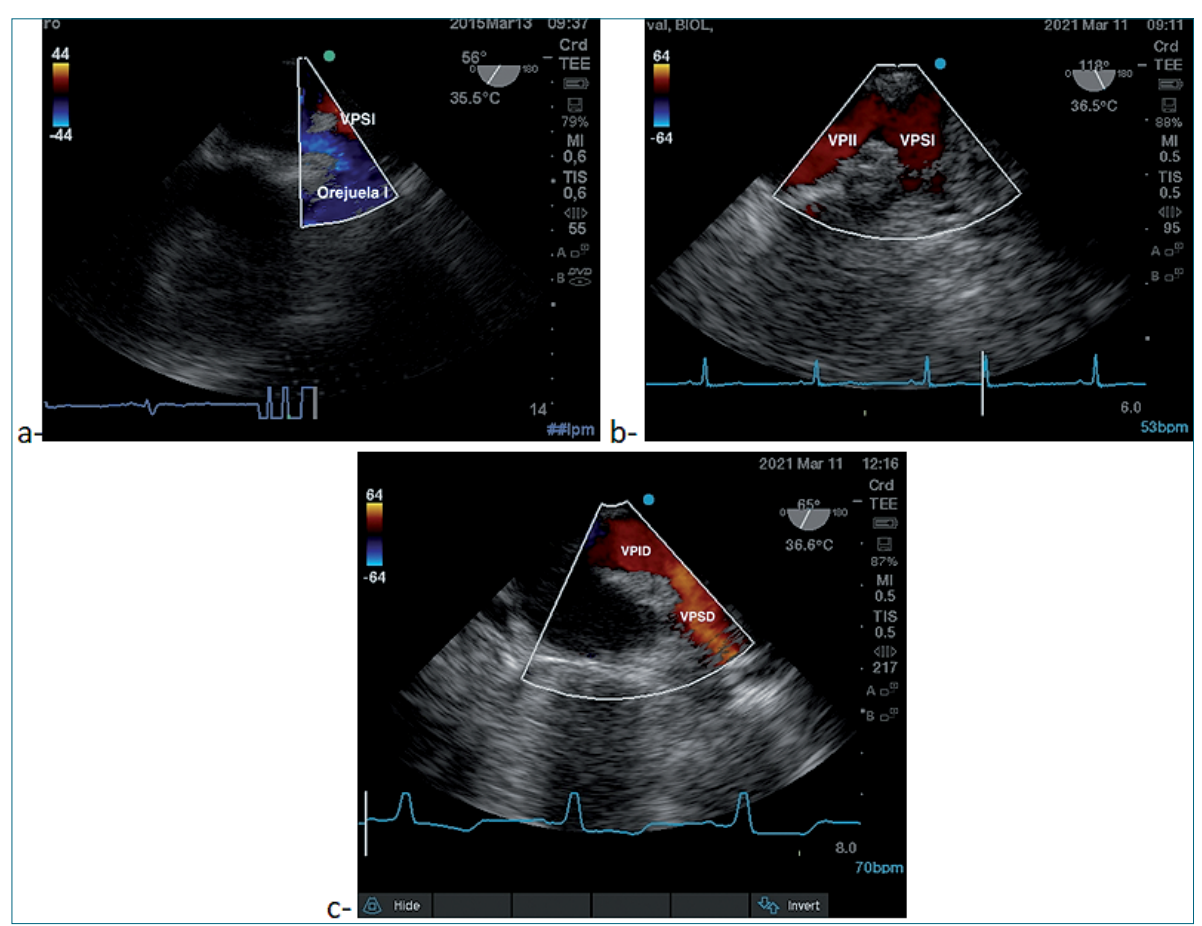

Figura 2. a- imagen $\mathrm{ME} 2$ cámaras $\left(56^{\circ}\right)$, doppler color azul apéndice Al, rojo VPSI, bME $118^{\circ}$ forma de $v$ invertida ambas VP izquierdas; c- ME $65^{\circ} \mathrm{VP}$ derechas. 
con la imagen centrada en la VPSI permite la visualización de la VPSI y VPII en forma invertida de v, (Figura 2b). La VPSD se identifica fácilmente girando la sonda hacia la derecha desde el apéndice de la aurícula izquierda o la vista bicava. Avanzar la sonda de 1 a $2 \mathrm{~cm}$ y girar más a la derecha permite visualizar VPID (Figura 2c)[4].

\section{El flujo venoso pulmonar normal}

Las VP son conductos de paredes delgadas y muy elásticos que permiten el mantenimiento de un volumen sistólico constante del ventrículo izquierdo $(\mathrm{VI})$ a pesar de la variación latido a latido del volumen sistólico del ventrículo derecho (VD). El flujo venoso pulmonar refleja el gradiente de presión entre las VP y la aurícula izquierda (Al). En consecuencia, las ondas Doppler en las VP reflejan las formas de onda de presión en la Al. El gradiente de presión VP-AI y, por lo tanto, el perfil de flujo de la VP dependen principalmente del ciclo cardíaco y la función

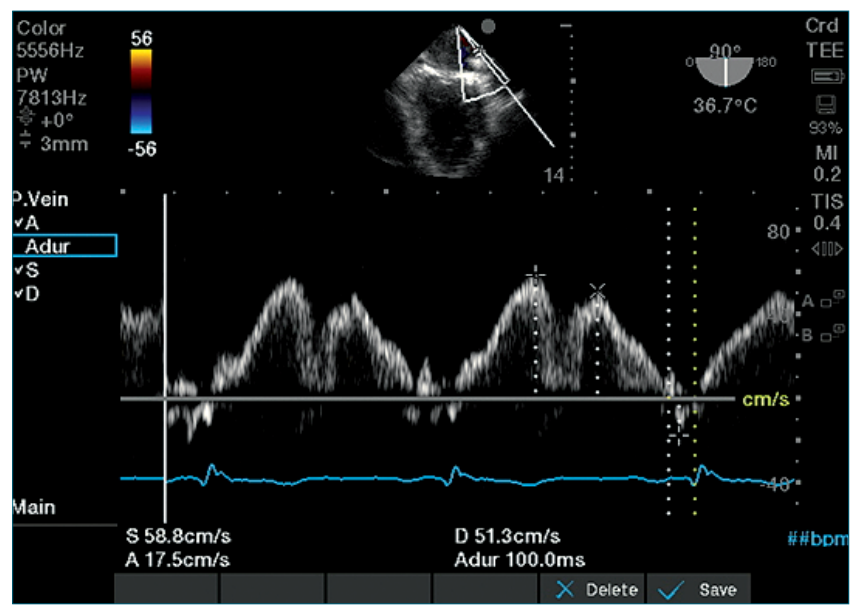

Figura 3. Flujo de velocidades normales de VP: ondas S/D y A, y duración de onda A.

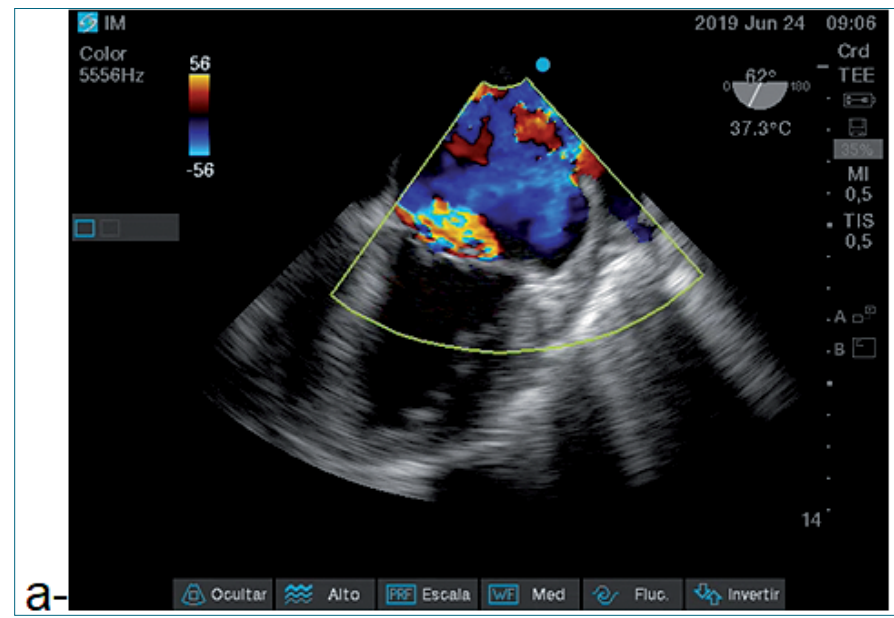

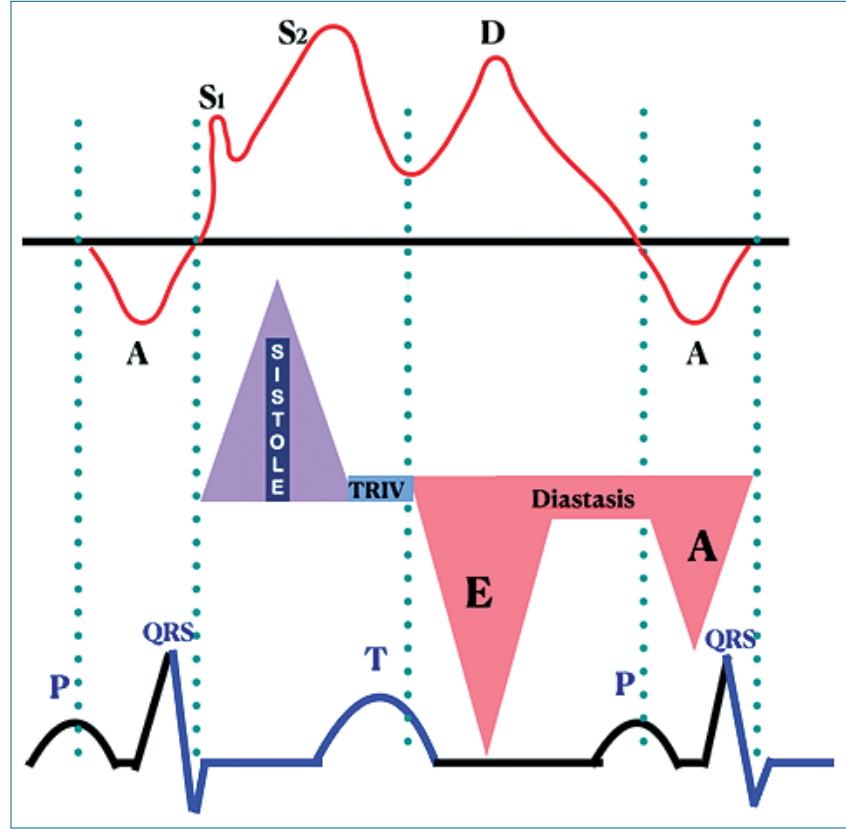

Figura 4. Relación de ondas VP con el flujo transmitral y el ciclo cardíaco.

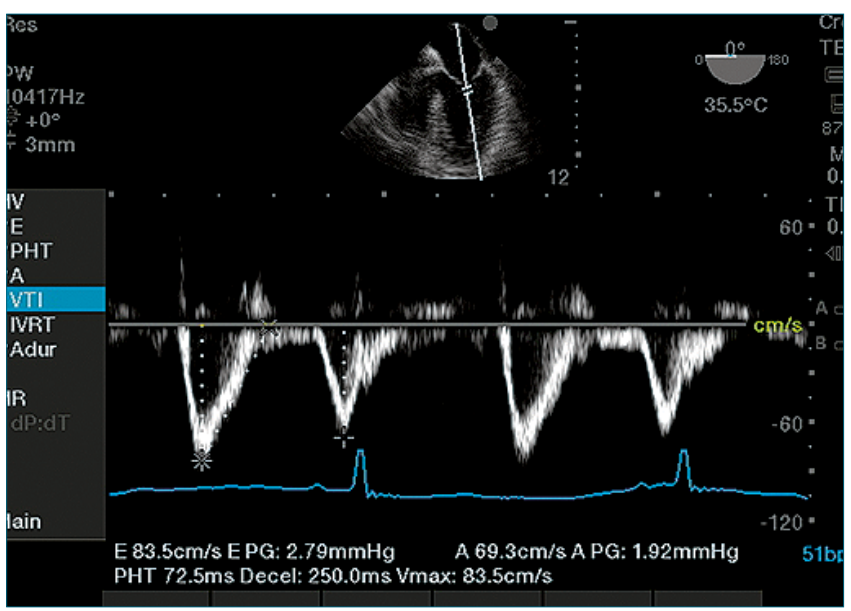

Figura 5. Flujo transmitral, doppler pulsado.

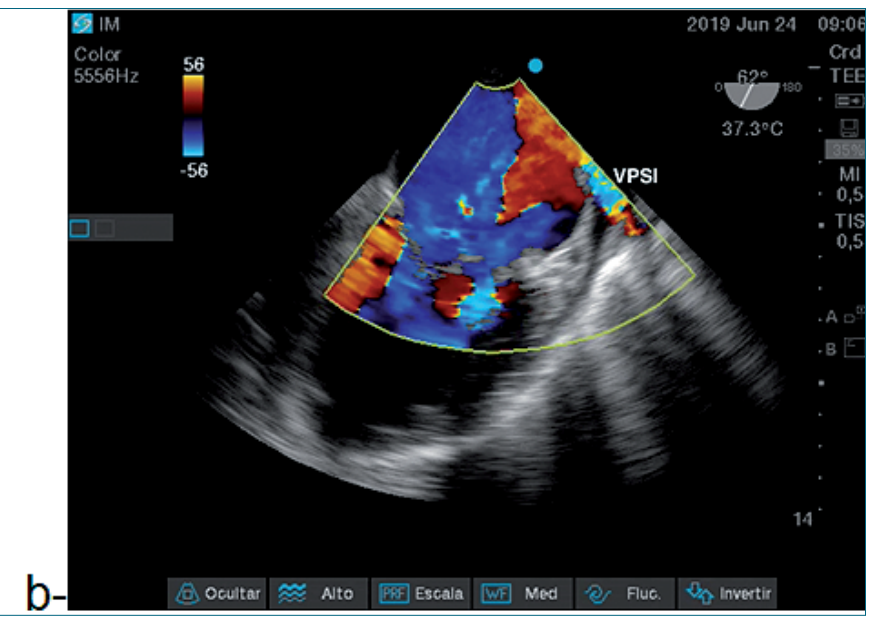

Figura 6. a- IM severa; b- reflujo de IM severa que llega a la VPSI (aliasing con doppler color). 
del corazón izquierdo. Además, están influenciados por el ciclo respiratorio, aunque en menor grado que el flujo en las venas cavas superior e inferior. Por lo tanto, el flujo de PV se altera en estados patológicos que afectan la función del corazón izquierdo o alteran el ritmo cardíaco[5].

La velocidad del flujo diastólico en la vena pulmonar está determinada por los mismos factores que influyen en las curvas de velocidad del flujo mitral[6]. El perfil de flujo normal en las VP muestra ondas fásicas y bidireccionales. Las fluctuaciones en la dirección y velocidad del flujo indican cambios en la presión de la Al. El flujo de VP refleja el llenado de Al a lo largo del ciclo cardíaco y el llenado del VI durante la diástole.

Se pueden identificar cuatro ondas Doppler distintas en las VP. Las dos primeras ondas anterógradas se observan en la sístole temprana (S1) y en la sístole media a tardía (S2), respectivamente, y ocurren durante la fase de reservorio de LA. Las ondas S1 y S2 a veces se ven fusionadas, formando una única onda sistólica. Estas dos ondas son discernibles por ETE en la mayoría de los casos. La tercera onda es anterógrada y se observa en la diástole temprana o media (onda D), que se produce durante la fase de conducto de la Al (Figura 3). La onda D está relacionada temporalmente con el descenso de la presión de Al y con la onda $\mathrm{E}$ transmitral en la imagen Doppler. La cuarta onda es retrógrada y se observa en la diástole tardía, onda A, que ocurre durante la fase de bombeo de Al. Está temporalmente relacionado con la onda A transmitral en la imagen Doppler (Figuras 4 y 5).

Las mediciones del doppler de las PV útiles incluyen la velocidad máxima de las ondas S, D y A, la relación de velocidad máxima S/D y la duración de la onda $A[7]$.

\section{Fisiología en la forma de onda VP doppler}

S1 se produce en respuesta a la caída de la presión de la Al provocada por el aumento del volumen auricular como resultado de la relajación auricular con un "efecto de succión" en la sangre. en la vasculatura pulmonar. Por tanto, S1 depende de la eficacia de la relajación de Al, por lo tanto, de la contractilidad auricular. S2 es el resultado de la propagación de la onda de eyección del VD a través de la vasculatura pulmonar. Además, S2 está determinado en parte por la distensibilidad de la $\mathrm{Al}$, que depende del desplazamiento sistólico el anillo mitral hacia el ápice. Por lo tanto, S2 depende del volumen sistólico del VD y de la función longitudinal del VI. La onda D es el resultado de la caída de la presión de la Al que sigue a la apertura de la válvula mitral (MV) y vaciado de la Al hacia el VI. Así, está determinada por el estado de relajación y distensibilidad del VI, y está influenciada por los mismos factores que afectan el llenado temprano de la onda E. Finalmente, la onda A es causada por la contracción auricular. Como la presión de Al excede la presión de VP, ocasiona una inversión del flujo en las VP. Por tanto, la onda A se ve afectada por la función contráctil de la Al y por la rigidez del VI[8].

La sincronización de las ondas S2 y D es relativamente fija durante el ciclo cardíaco debido a sus asociaciones con la sístole del VD y la relajación del VI, respectivamente. Por el contrario, la sincronización de las ondas A y S1 puede variar, ya que están influenciadas por factores variables como el ritmo cardíaco, la duración del intervalo PR y la relación entre la onda P y el complejo QRS.

\section{DOPPLER VP patológico}

Las alteraciones del perfil de flujo de VP ocurren en estados patológicos que involucran el miocardio, pericardio, VM o sistema de conducción del LV. Además, la estenosis de la VP y las anomalías congénitas que afectan a la aurícula izquierda provocan alteraciones en el Doppler VP. Algunas de estas condiciones conducen a cambios característicos en el perfil del flujo. A continuación, veremos alteraciones de las VP durante cirugía cardiaca más frecuente:

\section{Insuficiencia mitral}

ETE es un monitor indiscutible en todo tipo de cirugía cardíaca y de especial utilidad en cirugía de la válvula mitral[9].

El Doppler VP contribuye en la evaluación de la gravedad de la insuficiencia mitral (IM). El empeoramiento del grado de IM se asocia con una reducción progresiva de la velocidad pico de la onda S, debido a una disminución del componente S2, junto con un aumento en la velocidad de la onda D. Ocasionalmente, se encuentra una reversión holosistólica con la inversión de las ondas S1 y S2, especialmente cuando la IM es muy grave.

Mientras que la IM leve no altera el perfil de flujo de la VP en ausencia de patologías asociadas, en muchos pacientes con IM moderada o severa el flujo no se altera[3]. Y esto, dependiendo de la dirección y excentricidad del chorro de IM[10],[11].

Sobre la base de la información antes mencionada, el Doppler VP no debe utilizarse solo, sino junto con otros parámetros para ayudar a evaluar la gravedad de la IM. La ausencia de S invertida no excluye una IM grave, mientras que su presencia hace que la IM grave sea muy probable.

\section{Reparación de MV}

Uno de los procedimientos más modernos en pacientes con IM es la colocación de mitraclip. Datos recientes demuestran un valor para la evaluación intraprocedimiento del perfil doppler VP en el momento de la reparación percutánea de la VM para la insuficiencia mitral grave. Los datos de flujo de PV derivados de la ETE obtenidos en el laboratorio de cateterismo y que demuestran una relación S/D TVI baja $(<0,72)$ después de la implantación transcatéter de un clip de VM de borde a borde predicen una IM recurrente y un peor resultado a largo plazo[12]. La mejora en el perfil de flujo de PV de S invertida a señal atenuada $(S<D)$ y de señal atenuada a un patrón normal $(S>D)$ es un mejor predictor del resultado que el grado de repacación mitral y se asocia con un beneficio de supervivencia y una menor tasa de rehospitalización[13].

En la Figura 6 vemos aliasing de un alM tan severa que se refleja en la VPSI a $62^{\circ} \mathrm{ME}$, en la Figura 7a se observa una imagen de prolapso de VM que causa IM y en la Figura 7b el tratamiento quirúrgico (reemplazo de VM con válvula biológica). Y en las Figura 8a vemos la S invertida en el flujo doppler de VP y en la Figura 8b, luego de CEC y del reemplazo por la VM biológica, la normalización del flujo VP.

\section{Estenosis mitral}

La obstrucción significativa del flujo a través de la VM se asocia con una disminución en la velocidad de todas las formas de onda de PV (ondas S, D y A)[14].

En la Figura 9a vemos una estenosis mitral con doppler co- 


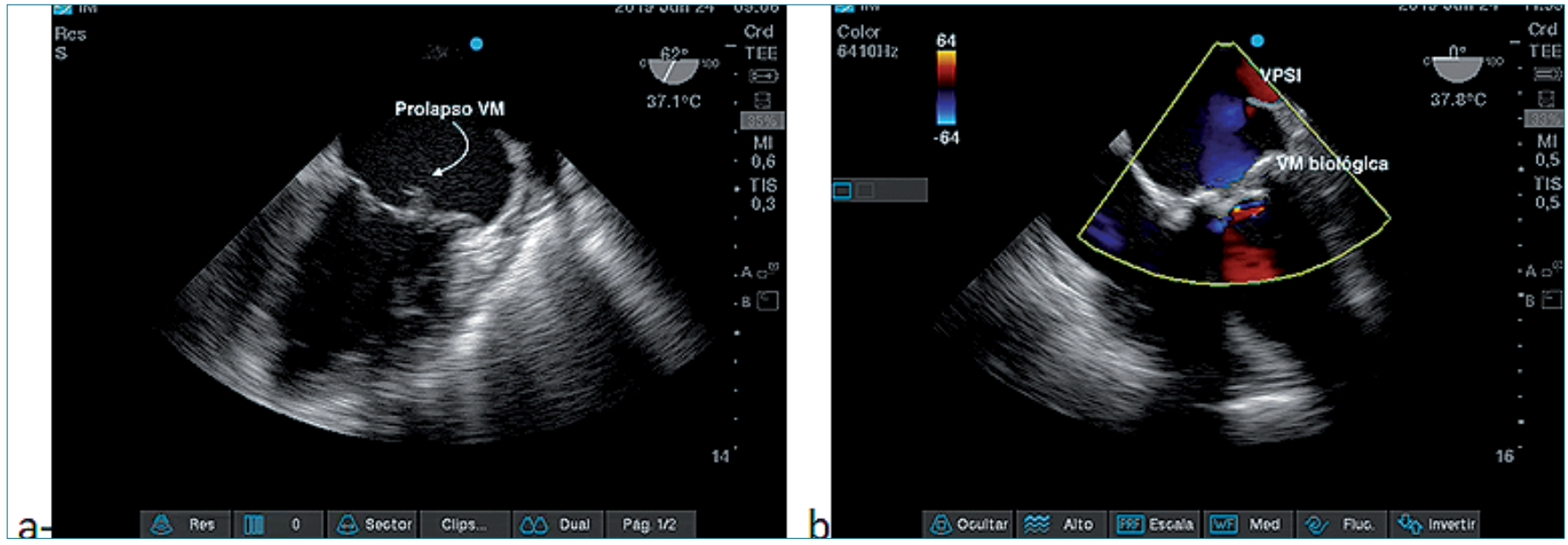

Figura 7. a- Imagen ME 2 cámaras, IM por prolapso VM; b- Reemplazo VM biológica sin aliasing VPSI.
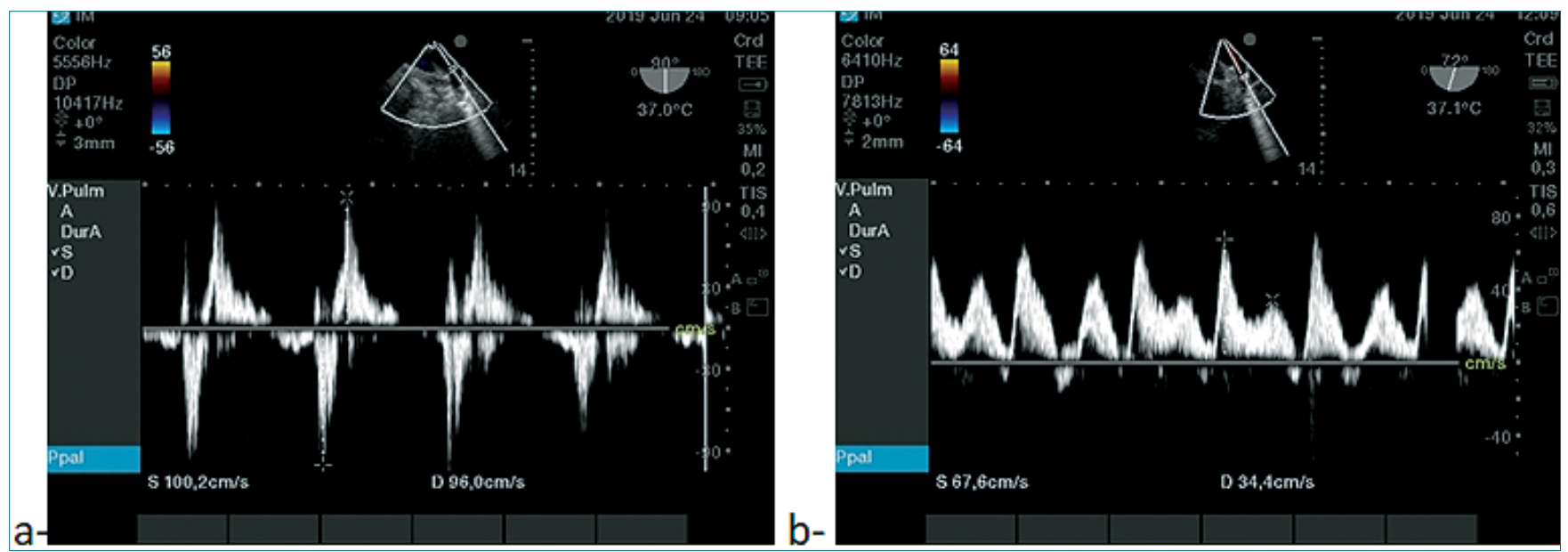

Figuras 8. a- IM severa onda S invertida; b- luego del reemplazo VM S positiva relación S/D normal mayor 1.
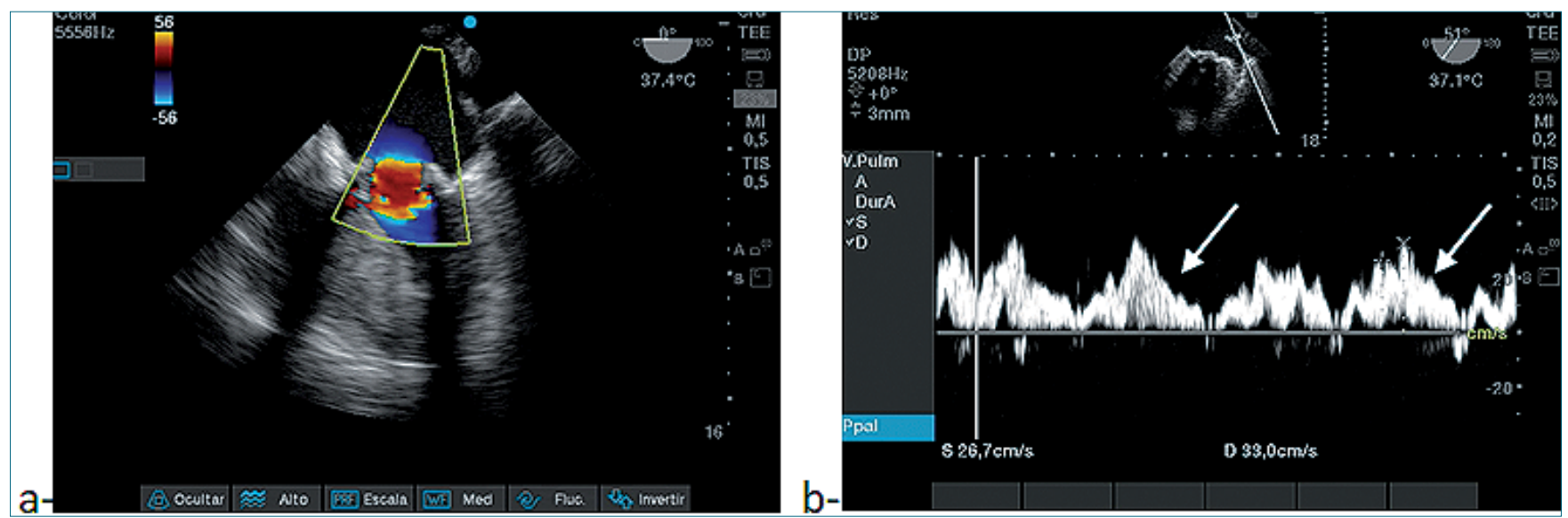

Figuras 9. a- Estenosis mitral, doppler color; b- Patrón doppler pulsado de VP, flechas indican desaceleración prolongada de la onda D. 
lor y en Figura 9b un tiempo medio de presión prolongado de la onda D que suele ser evidente, un reflejo de la disminución anormalmente lenta de la presión a través de la VM en la diástole temprana. Este patrón se refleja en un tiempo medio de presión anormalmente prolongado de la onda $\mathrm{E}$ transmitral (Figura 10). Además, ocasionalmente se observa una onda A prominente durante el ritmo sinusal, lo que indica una contracción forzada de LA contra la VM estenótica. Un patrón de PV similar que muestra una desaceleración prolongada de la onda $D$ se puede encontrar en el contexto de una relajación del VI alterada.

\section{Anomalías de la función diastólica VI}

La disfunción diastólica (DD) del ventrículo izquierdo (VI) es un marcador útil de morbilidad y mortalidad en pacientes de cirugía cardíaca. Es una enfermedad subestimada con un alto riesgo de descompensación aguda en el período perioperatorio[15].

La cirugía de revascularización coronaria y la cardíaca en general implican grandes alteraciones hemodinámicas. Los pacientes con isquemia miocárdica y enfermedad valvular con hi- pertrofia miocárdica son especialmente susceptibles de tener algún grado de DD, a pesar de tener fracción de eyección normal o levemente deteriorada.

Su identificación beneficia a los pacientes ofreciendo mejores intervenciones terapéuticas[16].

\section{Presión telediastólica del VI elevada}

La presión telediastólica del VI elevada (PTDVI) es uno de los primeros marcadores mensurables de disfunción diastólica que suele preceder al aumento de la presión media de la Al. La onda A del llenado ventricular y la onda A de la VP normalmente comienzan simultáneamente, y en sujetos sanos, sus duraciones son similares[17].

En el contexto de la PTDVI elevada, la presión diastólica de VI hace que la VM se cierre antes de que comience la relajación auricular y, por lo tanto, antes de que haya disminuido la presión de la Al. Este fenómeno da como resultado un reflujo de sangre más pronunciado hacia las VP y conduce a un A de VP más amplio que la A mitral (AM). Una duración de A VP-AM > 30 mseg predice un LVEDP alto con alta precisión y de manera independiente de la edad[18].

La diferencia A VP-AM ayuda a separar a los pacientes con

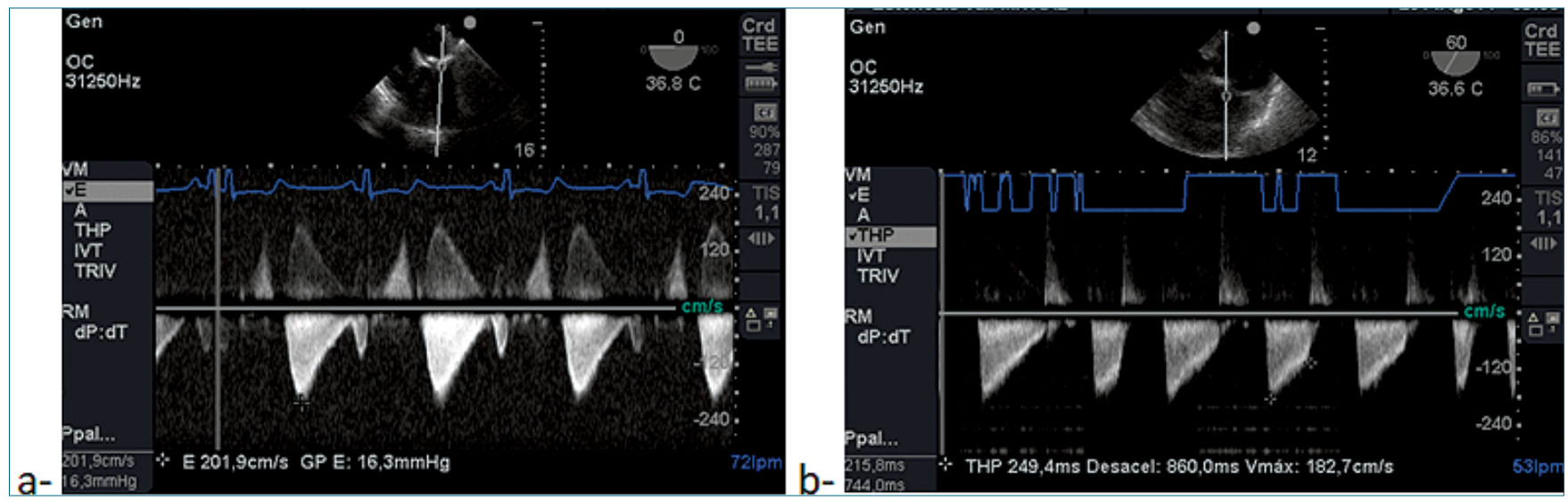

Figura 10. a- EM, elevado gradiente de la onda E (2m/s); b- Tiempo de hemipresión prolongado $(249 \mathrm{~m} / \mathrm{s})$, mediciones con doppler continuo de la VM.
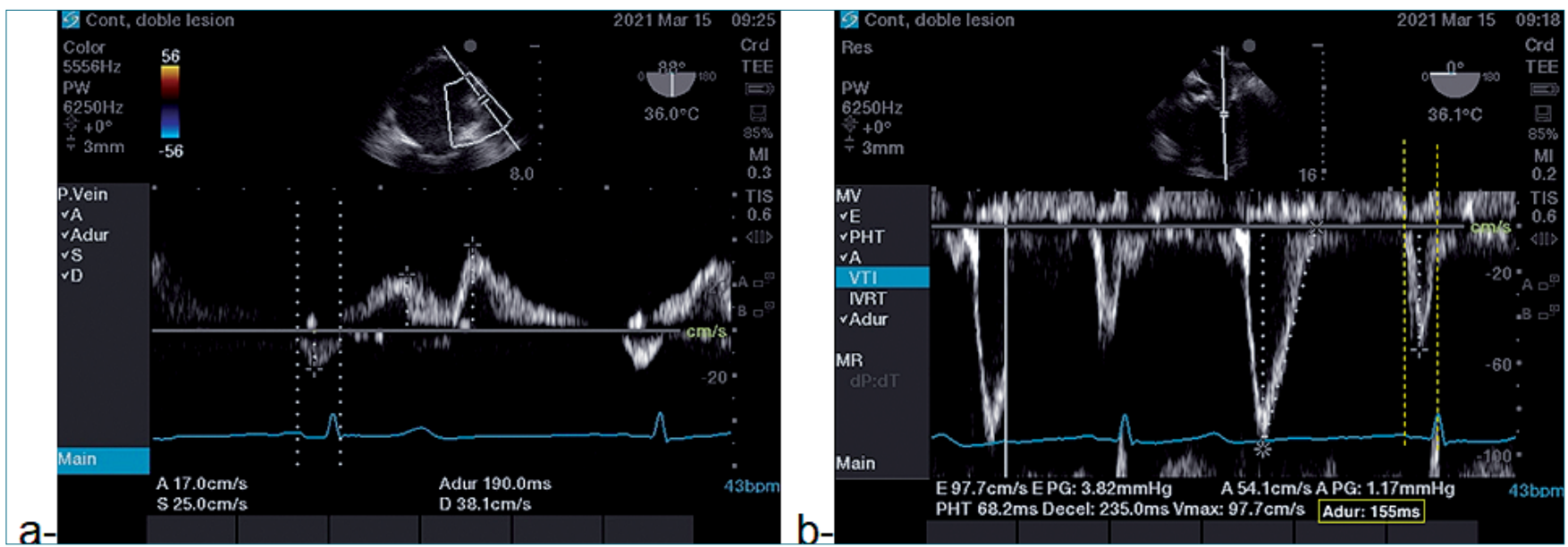

Figura 11. a- duración A VP; b- duración AM. Relación: A VP (190ms) - AM (155ms): 35 ms. 


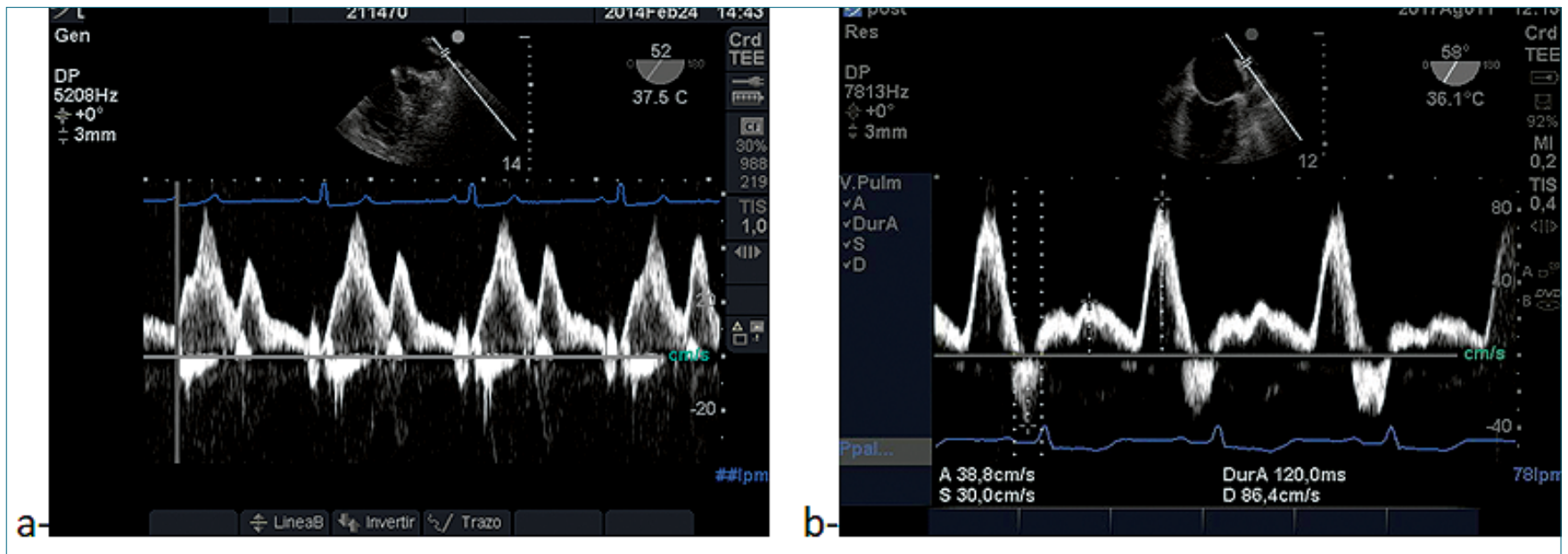

Figura 12. a- Patrón S/D mayor 1; b- Patrón $S / D<1$.

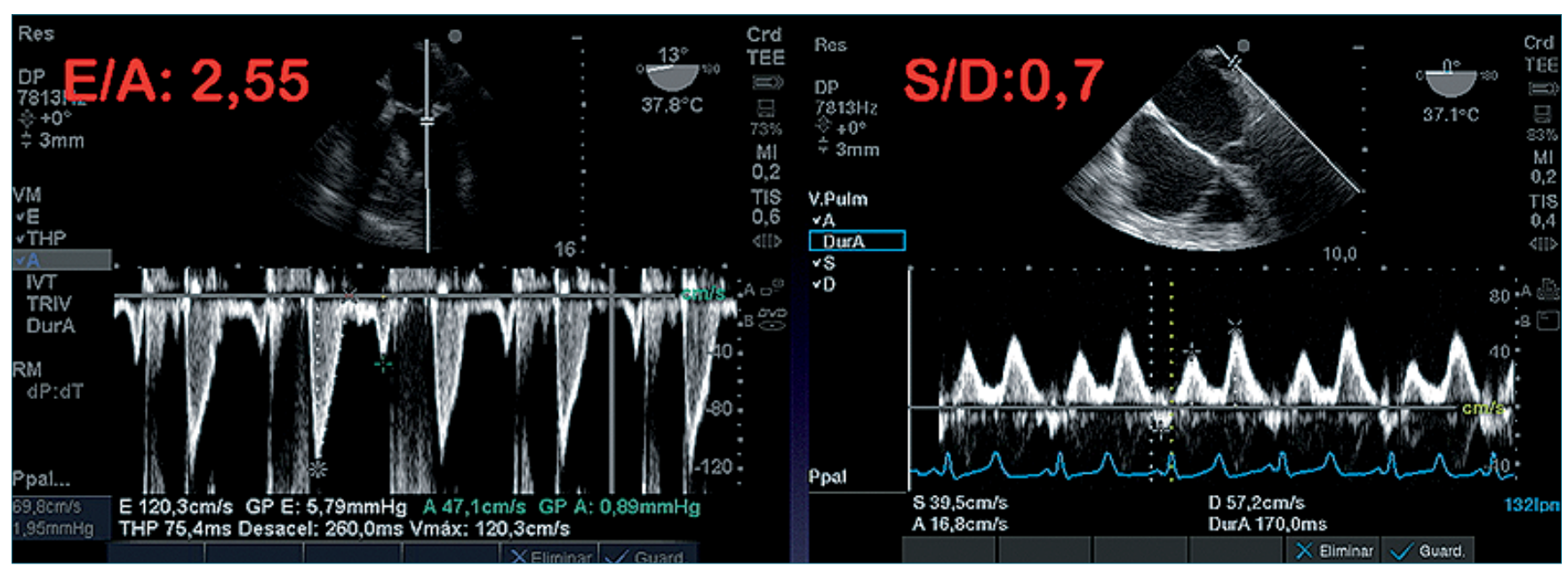

Figura 13. Relación del flujo transmitral con el de VP (doppler pulsado). En paciente con grado 3 de DD (E/A > 2 y S/D < 1).

patrón de relajación alterado en aquellos con presión de llenado normal y aquellos con PTDVI elevada y presión media de Al normal.

Es importante destacar que la evaluación de A VP no es factible durante la fibrilación auricular, debido a la pérdida de la contracción auricular.

\section{Presión Al elevada}

En adultos normales mayores de 40 años, la relación S/D es > 1 y muestra una relación inversa con la presión de Al. A medida que aumenta esta última, la velocidad de la onda $\mathrm{S}$ disminuye y el perfil Doppler VP muestra típicamente un patrón diastólico dominante $(S<D)$. La prominente onda $D$ refleja una onda E transmitral de alta velocidad, en sí misma una manifestación del alto gradiente de presión entre AI-VI. En las Figuras 12 a y b y Figura 13 observamos un paciente con DD, relación del flujo transmitral con Doppler pulsado (relación E/A > 1), en relación al flujo de VP (S/ D menor de 1)[19].

Como la onda S2 está influenciada por la contractilidad del $\mathrm{VI}$ y el volumen sistólico, una relación $\mathrm{S} / \mathrm{D}<1$ es un marcador de presión elevada de Al en pacientes con fracción de eyección de VI (FEVI) reducida y/ o gasto cardíaco bajo.

En individuos con FEVI "normal", el cociente S/D no es un marcador sensible de presión de llenado elevada, debido al movimiento anular mitral conservado; una relación S/ D > 1 (Figura 12 a) "no excluye" la presión Al elevada en el contexto de FEVI conservada.

Cuando está presente, una relación S/ D baja $<1$ (Figura 12 b) es un "predictor independiente" de supervivencia y eventos cardiovasculares futuros en individuos con FEVI preservada y proporciona información pronóstica más allá de la clasificación tradicional de la función diastólica[20].

La relación $S / D<1$ no predice la presión de la Al elevada en $<$ de 40 años, ni en el contexto de fibrilación auricular, insuficiencia mitral grave y miocardiopatía hipertrófica. 


\author{
Valor pronóstico de los hallazgos en el flujo venoso pul- \\ monar:
}

Los parámetros Doppler VP, tienen valor pronóstico y son predictores de eventos cardíacos adversos importantes luego de la cirugía cardíaca, incluida la hospitalización prolongada por fibrilación ventricular, edema pulmonar por insuficiencia cardíaca, reingreso hospitalario y tasas de mortalidad más altas en pacientes con mayor grado de DD[21].

Los datos obtenidos de los estudios ecocardiográficos se utilizan a diario para orientar la toma de decisiones clínicas. La observación con ETE de las VP es simple y se puede tener en segundos en la mayoría de los pacientes. La conformación de sus ondas puede ir orientando acerca del estado hemodinámico del corazón izquierdo, mientras vamos logrando interrogar otros aspectos, además de las VP.

Si a estos valores de Doppler VP y mitral le sumamos la presión sistólica de la arteria pulmonar interrogada a través del reflujo tricuspídeo y analizamos en 2D el patrón pulmonar y observamos líneas $A$ o presencia de líneas $B$ tendremos un cuadro clínico completo acerca del grado de DD y de la repercusión o no cardiopulmonar de la enfermedad.

\section{Conclusión}

La valoración del Doppler VP debería realizarse de rutina en toda cirugía cardíaca.

Dada la proximidad de la sonda de ETE con la Al podemos lograr imágenes óptimas. Debido además, a la alineación ideal de la VP superior izquierda para ser interrogada con el Doppler pulsado. Anestesiólogos e intensivistas que utilizamos ETE habitualmente interrogando el Doppler de VP podemos sumar valiosa información en el pronóstico de los pacientes críticos. Aunque no se deben utilizar los resultados del perfil Doppler VP como los únicos parámetros para fines de diagnóstico, su uso en combinación con otros parámetros de Doppler pulsado mitral, suma valiosa información hemodinámica adicional respecto del estado del corazón izquierdo.

\section{Referencias}

1. Zoghbi WA, Adams D, Bonow RO, et al. Recommendations for noninvasive evaluation of native valvular regurgitation: a report from the American Society of Echocardiography developed in collaboration with the Society for Cardiovascular Magnetic Resonance. J Am Soc Echocardiogr 2017; 30:303-71. https://doi. org/10.1016/j.echo.2017.01.007

2. Sohns $C$, Vollmann $D$, Luethje $L$, et al. MDCT in the diagnostic algorithm in patients with symptomatic atrial fibrillation. World $J$ Radiol 2011; 3:41-6. https://doi.org/10.4329/wjr.v3.i2.41

3. Castello R, Pearson AC, Lenzen $P$, et al. Evaluation of pulmonary venous flow by transesophageal echocardiography in subjects with a normal heart: comparison with transthoracic echocardiography. J Am Coll Cardiol 1991; 18:65-71. https://doi. org/10.1016/S0735-1097(10)80219-0

4. Hahn RT, Abraham T, Adams MS, et al. Guidelines for performing a comprehensive transesophageal echocardiographic examination: recommendations from the American Society of Echocardiography and the Society of Cardiovascular Anesthesiologists. J
Am Soc Echocardiogr 2013; 26:921-64. https://doi.org/10.1016/j. echo.2013.07.009

5. Tabata T, Thomas JD, Klein AL. Pulmonary venous flow by Doppler echocardiography: revisited 12 years later. J Am Coll Cardiol 2003; 41:1243-50. https://doi.org/10.1016/S07351097(03)00126-8

6. Nishimura RA, Abel MD, Hatle LK, Tajik AJ. Relation of pulmonary vein to mitral flow velocities by transesophageal Doppler echocardiography. Effect of different loading conditions. Circulation 1990; 81:1488-97. https://doi.org/10.1161/01.CIR.81.5.1488

7. Fadel B.M., Pibarot P., Kazzi B.E., et al. Spectral Doppler Interrogation of the Pulmonary Veins for the Diagnosis of Cardiac Disorders: A Comprehensive Review. J Am Soc Echocardiogr. 2021 34(3):223-236. https://doi.org/10.1016/j.echo.2020.09.012

8. Smiseth OA, Thompson CR, Lohavanichbutr $\mathrm{K}$, Ling $\mathrm{H}$, et al. The pulmonary venous systolic flowpulse-its origin and relationship to left atrial pressure. J Am Coll Cardiol 1999; 34:802-9. https://doi. org/10.1016/S0735-1097(99)00300-9

9. Nicoara A, Skubas N, Ad N, et al. Guidelines for the Use of Transesophageal Echocardiography to Assist with Surgical DecisionMaking in the Operating Room: A Surgery-Based Approach From the American Society of Echocardiography in Collaboration with the Society of Cardiovascular Anesthesiologists and the Society of Thoracic Surgeons. J Am Soc Echocardiogr 2020; 33:692-734 https://doi.org/10.1016/j.echo.2020.03.002

10. Mark JB, Ahmed SU, Kluger R, et al. Influence of jet direction on pulmonary vein flow patterns in severe mitral regurgitation. Anesth Analg 1995; 80:486-91. https://doi. org/10.1213/00000539-199503000-00009

11. Klein AL, Bailey AS, Cohen Gl, et al. Importance of sampling both pulmonary veins in grading mitral regurgitation by transesophageal echocardiography. J Am Soc Echocardiogr 1993; 6:115-23. https://doi.org/10.1016/S0894-7317(14)80481-1

12. Ikenaga $H$, Yoshida J, Hayashi A, et al. Usefulness of intraprocedural pulmonary venous flow for predicting recurrent mitral regurgitation and clinical outcomes after percutaneous mitral valve repair with the MitraClip. JACC Cardiovasc Interv 2019;12:14050. https://doi.org/10.1016/j.jcin.2018.09.034

13. Corrigan FE III, Chen JH, Maini A, et al. Pulmonary venous waveforms predict rehospitalization and mortality after percutaneous mitral valve repair. JACC Cardiovasc Imaging 2019; 12:1905-13. https://doi.org/10.1016/j.jcmg.2018.07.014

14. Klein AL, Bailey AS, Cohen GI, Stewart WJ, Husbands K, Pearce $G L$, et al. Effects of mitral stenosis on pulmonary venous flow as measured by Doppler transesophageal echocardiography. Am J Cardiol 1993;72 :66-72. https://doi.org/10.1016/00029149(93)90221-W

15. Nagueh S. F., Smiseth O. A., Appleton C.P., et al. Recommendations for the Evaluation of Left Ventricular Diastolic Function by Echocardiography: An Update from the American Society of Echocardiography and the European Association of Cardiovascular Imaging. JAm Soc Echocardiogr 2016;29:277-314. https://doi. org/10.1016/j.echo.2016.01.011

16. Dominella F.G., De Brahi J.I., Longo S. 2018. Presentación Póster $45^{\circ}$ en el Congreso Argentino de Anestesiología.

17. Oh JK, Appleton CP, Hatle LK, et al. The noninvasive assessment of left ventricular diastolic function with twodimensional and Doppler echocardiography. J Am Soc Echocardiogr 1997; 10:24670. https://doi.org/10.1016/S0894-7317(97)70062-2

18. Yamamoto K, Nishimura RA, Burnett JC Jr., Redfield MM. As- 
sessment of left ventricular end-diastolic pressure by Doppler echocardiography: contribution of duration of pulmonary venous versus mitral flow velocity curves at atrial contraction. J Am Soc Echocardiogr 1997; 10:52-9. https://doi.org/10.1016/S08947317(97)80032-6

19. Kuecherer HF, Muhiudeen IA, Kusumoto FM, et al. Estimation of mean left atrial pressure from transesophageal pulsed Doppler echocardiography of pulmonary venous flow. Circulation 1990; 82:1127-39. https://doi.org/10.1161/01.CIR.82.4.1127

20. Buffle E, Kramarz J, Elazar E, et al. Added value of pulmonary ve- nous flow Doppler assessment in patients with preserved ejection fraction and its contribution to the diastolic grading paradigm. Eur Heart J Cardiovasc Imaging 2015; 16:1191-7. https://doi. org/10.1093/ehjci/jev126

21. Ren $X, N a B$, Ristow $B$, Whooley MA, Schiller NB. Usefulness of diastolic dominant pulmonary vein flow to predict hospitalization for heart failure and mortality in ambulatory patients with coronary heart disease (from the Heart and Soul Study). Am J Cardiol 2009; 103:482-5. https://doi.org/10.1016/j.amjcard.2008.10.024 